\title{
The effects of external and internal focus of attention on motor learning and promoting learner's focus
}

\author{
Saeed Ghorbani ${ }^{1}$, Amir Dana ${ }^{2}$, Zynalabedin Fallah \\ 1 Department of Physical Education and Sport Sciences, Aliabad Katoul Branch, Islamic Azad University, Aliabad Katoul, \\ Iran; ${ }^{2}$ Department of Physical Education and Sport Sciences, Tabriz Branch, Islamic Azad University, Tabriz, Iran; \\ ${ }^{3}$ Department of Physical Education and Sport Sciences, Gorgan Branch, Islamic Azad University, Gorgan, Iran
}

\section{Summary}

Study aim: External focus of attention is considered as a critical factor in the OPTIMAL theory of motor learning. This theory proposes that external focus of attention facilitates motor performance and learning because it promotes focusing on the task goal. However, the effects of external focus of attention on focusing on the task goal are not well understood. The aim of this study was, therefore, to investigate the effects of an external focus of attention versus an internal focus of attention on motor learning and promoting focus of the learner on the task goal.

Material and methods: Thirty-six right-handed male students (mean age $21.16 \pm 1.85$ years old) with no prior experiences with the motor task were randomly assigned to three groups: external focus, internal focus, and control groups. Participants were asked to throw darts at a target during an acquisition phase (10 blocks of six trials each) and during subsequent retention and transfer tests. Throwing accuracy and focus on the task goal were measured as dependent variables. Analysis of variance (ANOVA) with repeated measures as well as a one-way ANOVA was used to analyze the differences in accuracy scores between groups during the acquisition phase as well as retention and transfer tests, respectively. The significance level was set at $p<.05$. The author supervised all phases of the experiment.

Results: The results showed that adopting an external focus promoted a focus on the task goal and resulted in significantly better motor learning than adopting an internal focus and control conditions $(p<0.05)$.

Conclusions: The findings of the present study provided support for the propositions of the OPTIMAL theory and showed that adopting an external focus of attention promotes focus of the learner on the task goal. The results are discussed in terms of benefits of external focus instructions for facilitating motor learning and goal-action coupling.

\section{Keywords: OPTIMAL theory - External focus of attention - Goal-action coupling - Motor learning}

\section{Introduction}

An interesting topic for researchers in the field of motor learning is to understand the practice conditions that facilitate motor performance and learning. Wulf and Lewthwaite [27] proposed the OPTIMAL (Optimizing Performance through Intrinsic Motivation and Attention for Learning) theory of motor learning and integrated the evidence from several lines of research that demonstrate important roles for motivation and attentional focus in motor performance and learning [27]. According to the OPTIMAL theory, practice conditions that facilitate motor performance and learning are (a) enhanced expectancies, (b) autonomy support and (c) external focus of attention.
Enhanced expectancies and autonomy support are considered as motivational factors and have been well documented by previous research $[4-6,8-12,14,17,20,25$, 29, 30]. Moreover, external focus of attention is considered as an attentional factor, which optimizes performance and learning a new motor skill [27]. Although the effects of adopting an external focus of attention on motor performance and learning are well documented [1, 2, 13, 16, $18,19,21-24,26,28,29]$, mechanisms underlying this issue are not well understood. Therefore, the present study was designed to further investigate the effects of adopting an external focus of attention on motor learning as well as promoting focus of the learner on the task goal as an underlying mechanism for the effects of external focus of attention on motor learning [27]. 
In the literature, external focus of attention refers to focusing on the intended movement effect such as a dartboard or the path of the dart in dart throwing [27]. Several studies have shown beneficial effects for an external focus of attention in comparison to an internal focus of attention, i.e., focusing on the hand while throwing a dart $[1,2,13$, $16,18,19,21-24,26,28,29]$. For instance, Wulf and Su [28] asked novices to hit golf balls into a circular target. Participants in the internal focus of attention were instructed to focus on the swinging motion of their arms while those in the external focus of attention were instructed to focus on the pendulum-like motion of the club. The results revealed that the external focus group had significantly higher accuracy scores than the internal focus group in the retention test. In addition, Wulf et al. [26] replicated these results by using a soccer throw-in task in children.

According to the OPTIMAL theory, adopting an external relative to an internal focus plays a dual role by (a) directing attention to the task goal and (b) reducing a focus on the self. Thus, an external focus is an important contributor to goal-action coupling (27). Wulf and Lewthwaite [27] assumed that goal-action coupling is an underlying mechanism for motor learning following adopting an external focus. In a recent study, Abdollahipour et al. [2] investigated this proposition. In this study, the children were asked to roll a bowling ball towards 10 pins. Participant performed 8 trials under external focus (path of the ball), internal focus (hand), or control conditions. The participants were also asked to respond to a verbal report following eight trials to serve as a manipulation check for treatment effectiveness (the questions were 1 . What did you focus on? and 2. How much did you focus on it?). The results showed that external focus of attention led to a significantly better motor performance than internal focus of attention. In addition, participants' responses to the manipulation check showed that they followed the external and internal foci instructions to a large extent and ratings of the intensity of participants' foci were very high. No retention test was included in this study. Thus, the question remained unclear whether the learners could maintain focus on the task goal when no external focus instructions were available (e.g., in retention or transfer tests).

The purpose of the present experiment was, therefore, to extend the previous results and further examine the effects of adopting an external focus versus adopting an internal focus of attention on motor performance, learning, and focusing on the task goal as an underlying mechanism for the effects of external focus of attention on motor learning. In order to examine whether adopting an external focus resulted in focusing on the task goal, we applied a manipulation check similar to what was used by Abdollahipour et al. [1, 2]. Furthermore, we added retention and transfer tests to the study in order to examine whether the learners maintain their focus on the task goal when there are no focus instructions, i.e., in retention and transfer tests. According to the OPTIMAL theory, we hypothesized that the participants who adopted an external focus of attention would show better motor learning than those who adopted an internal focus of attention and control condition in performance, retention, and transfer tests. Moreover, we hypothesized that adopting an external focus of attention leads to focusing on the task goal during the acquisition, retention and transfer tests.

\section{Material and methods}

\section{Participants}

According to previous research $[2,14,18], 36$ male undergraduate students (mean age $21.16 \pm 1.85$ years old) participated voluntarily in this study and were randomly allocated to three groups: external focus of attention (EXT), internal focus of attention (INT), and control groups. The participants were right-handed with no previous experiences with the experimental task and were recruited from general physical education classes during winter semester $2018 / 19$. The protocol was performed in accordance with the Declaration of Helsinki and was approved by the university's institutional review board. The participants gave written consents.

\section{Motor task}

An underarm dart throwing was selected as the motor task. This task was originally used by Al-Abood, Davids, and Bennett [3]. The aim of the task was to score as many points as possible by aiming the dart towards a target dartboard. The target was a standard dartboard modified for the outcome scoring system of the study. It contained 10 concentric circles. The bullseye had a diameter of 2.25 $\mathrm{cm}$, with each other circle increasing by $2.25 \mathrm{~cm}$ in radius. To provide outcome scores as a dependent variable, the bullseye was awarded 10 points, with each concentric circle radiating out from the bullseye decreasing by one point so that the outermost circle was worth only one point. The target was placed on the floor $3 \mathrm{~m}$ away from a throwing line [3]. A regular (Unicorn) dart was used.

\section{Procedure}

Participants were tested individually in two days. Prior to data collection, participants were given general information of the experimental process and then completed a questionnaire to obtain information such as age, laterality, and previous experiences in darts. To create groups that would be more similar at baseline we recruited only bachelor students, right handed participants, and those who had no previous experience in dart throwing. Finally, participants were given brief instruction on dart 
throwing consisting of holding the dart in the hand and throwing it towards the dartboard. During the acquisition phase, participants performed ten blocks of 6 trials, and one day later they performed retention and transfer (with left hand) tests consisting of 10 trials. Participants in the EXT group received instruction focusing on the bullseye while throwing the dart. Those in the INT group received instruction that focused on their hand while throwing the dart. Participants in the control group received no focus instruction. Participants received 10 seconds to perform a throw. KR was not presented during the experiment. Before each block, the experimenter reminded the participants to maintain their respective focus. All the participants performed a manipulation check following completing the acquisition, retention and transfer tests. In order to measure the kind of focus the participants were asked, "What did you focus on?", and, then, to measure intensity of focus, they were asked to indicate on a Likert scale from 1 (not at all) to 7 (very much) "How much did you focus on it?" [2].

\section{Data analysis}

Accuracy scores during the acquisition phase were analyzed by analysis of variance (ANOVA) with repeated measures. A one-way ANOVA was used to analyze the differences in accuracy scores between groups in the retention and transfer tests. Differences between groups in the intensity of attentional focus in the manipulation check during the acquisition, retention and transfer tests were analyzed by a one-way ANOVA. The Tukey test was used as a post-hoc test. The significance level was set at $\alpha<0.05$.

\section{Results}

\section{Accuracy scores}

Analysis of the first block showed no significant differences between experimental groups at the beginning of practice, $\left(F_{2,33}=3.07, p>0.05\right)$. During the acquisition phase, the main effect for Group was not significant, $\left(F_{2,33}=0.84, p>0.05\right)$. Moreover, the main effect for Block was significant, $\left(F_{9,297}=2.81, p=0.003, \eta^{2}=0.07\right)$, however, the Group x Block interaction was not significant, $\left(F_{18,297}=1.32, p>0.05\right)$. A main effect for Block means that all groups improved their accuracy scores during the acquisition phase (see Figure 1). The results showed a significant main effect for Group in the retention test, $\left(F_{2,33}=4.93, p=0.019, \eta^{2}=0.21\right)$. Post hoc analysis revealed that external focus instruction resulted in better accuracy scores than internal focus instruction $(p<0.05)$. No significant difference was observed between internal focus instruction and control condition $(p>0.05)$. In addition, there were no significant differences between groups in the transfer test, $\left(F_{2,33}=0.36, p>0.05\right)$.

\section{Manipulation check}

The results of the manipulation check revealed that adopting an external focus of attention promoted focusing on the task goal whereas adopting an internal focus did not promote focusing on the task goal (see Table 1). The results of ANOVA showed no significant difference between groups in intensity of attentional focus in the acquisition phase $\left(F_{2,33}=0.64, p>0.05\right)$, retention $\left(F_{2,33}=1.27\right.$, $p>0.05)$ and transfer $\left(F_{2,33}=0.53, p>0.05\right)$ tests.

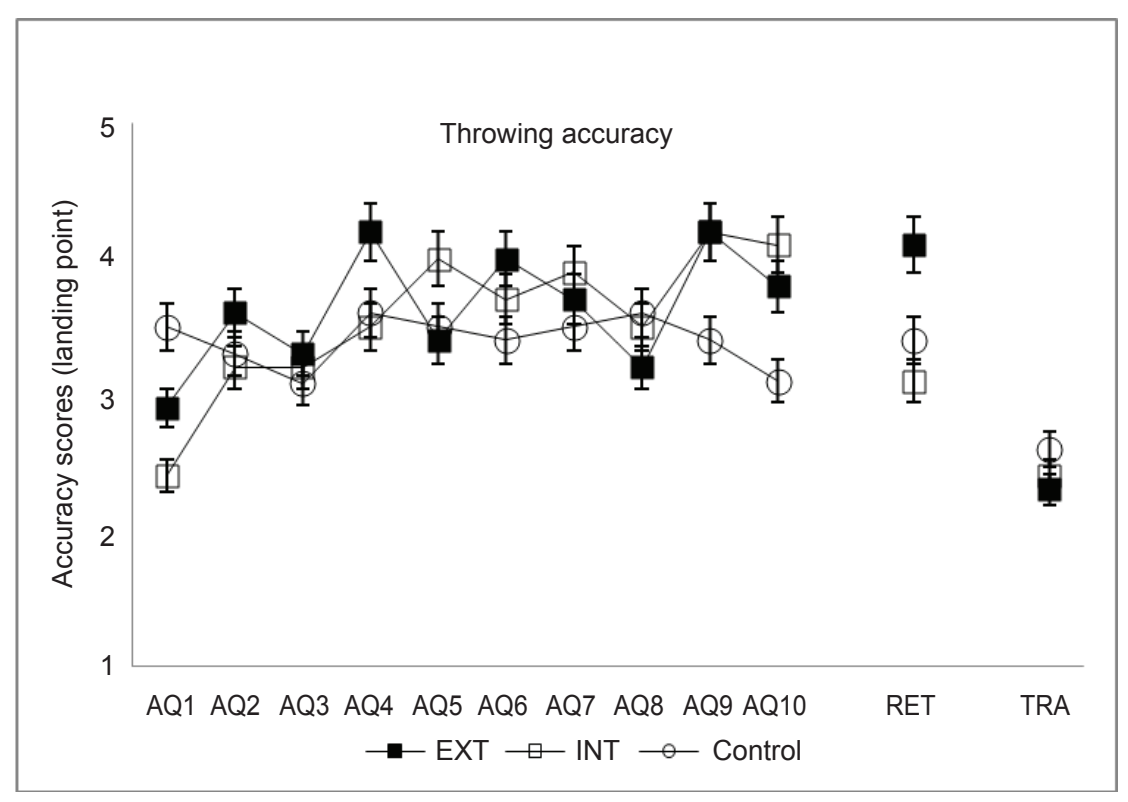

Fig. 1. Accuracy scores for the experimental groups across acquisition blocks and retention and transfer tests (EXT: external focus; INT: internal focus; AQ: acquisition; RET: retention; TRA: transfer) 
Table 1. Participants' responses to the questions of the manipulation check following acquisition, retention and transfer tests and across groups

\begin{tabular}{|c|c|c|c|c|c|c|c|c|c|c|c|c|c|}
\hline & & \multicolumn{6}{|c|}{ Externally focus reported } & \multicolumn{6}{|c|}{ Internally focus reported } \\
\hline & & \multicolumn{3}{|c|}{ Dartboard } & \multicolumn{3}{|c|}{ Path of the dart } & \multicolumn{3}{|c|}{ Hand } & \multicolumn{3}{|c|}{ Fingers } \\
\hline & & $\mathrm{Aq}$ & Ret & Tra & $\mathrm{Aq}$ & Ret & Tra & $\mathrm{Aq}$ & Ret & Tra & $\mathrm{Aq}$ & Ret & Tra \\
\hline \multirow[t]{2}{*}{$\mathrm{EF}$} & What & $75 \%$ & $58.3 \%$ & $50 \%$ & $25 \%$ & $41.7 \%$ & $16.7 \%$ & - & - & $33.3 \%$ & - & - & - \\
\hline & Intensity & 6.1 & 6.5 & 5.5 & 7 & 6.4 & 7 & - & - & 6.2 & - & - & - \\
\hline \multirow[t]{2}{*}{ IF } & What & $25 \%$ & $8.3 \%$ & - & - & - & - & $58.3 \%$ & $66.7 \%$ & $83.3 \%$ & $16.7 \%$ & $25 \%$ & $16.7 \%$ \\
\hline & Intensity & 6 & 6 & - & - & - & - & 6.2 & 6.2 & 6.3 & 5.5 & 6 & 6.5 \\
\hline \multirow[t]{2}{*}{$\mathrm{C}$} & What & $33.3 \%$ & $33.3 \%$ & $16.7 \%$ & $25 \%$ & $16.7 \%$ & - & $33.3 \%$ & $33.3 \%$ & $58.3 \%$ & $8.3 \%$ & $16.7 \%$ & $25 \%$ \\
\hline & Intensity & 6.7 & 6.2 & 5.5 & 6.3 & 7 & - & 6.2 & 6.2 & 6.2 & 6 & 7 & 6.6 \\
\hline
\end{tabular}

* EF - external focus of attention group; IF - internal focus of attention group; C - control group; Aq - acquisition phase; Ret - retention test; Tra - transfer test.

\section{Discussion}

An important factor in the OPTIMAL theory is external focus of attention [26]. The purpose of this study was to extend previous findings and further examine the effects of adopting an external focus of attention on motor learning and promoting focus on the task goal by using a sport skill as a to-be-learnt motor task. It was hypothesized that: 1) the participants who adopted an external focus of attention would show better motor learning than those who adopted an internal focus of attention and control condition in performance, retention, and transfer tests, 2) adopting an external focus of attention would lead to focusing on the task goal during the acquisition, retention and transfer tests.

The results of this study were in accordance with our hypotheses. The results demonstrated that adopting an external focus of attention led to significantly higher accuracy scores in the delayed retention test than an internal focus of attention, but it did not result in superior performance in the acquisition phase or transfer test. That is, adopting an external focus of attention resulted in a relatively permanent, or learning, effect in comparison to adopting an internal focus of attention. These results are in accordance with previous research $[1,2,13,16,18,19,21-24,26,28]$ and confirm the propositions of the OPTIMAL theory of motor learning [27] by indicating that adopting an external focus of attention is clearly beneficial for motor learning.

The results of the manipulation check appeared to be interesting. Generally, the results revealed that both the external and internal focus of attention groups followed the instructions given by the experimenter. In the acquisition phase and the retention test, all participants of the external focus group reported that they focused on the dartboard or path of the dart. However, in the transfer test $33.3 \%$ of them reported that they focused on their hand while throwing the dart. A possible reason for these finding in the transfer test might be because they are needed to throw the dart with their non-dominant hand during the transfer test, they focused on their hand to freeze more degrees of freedom and subsequently have more control on the dart throwing $[6,15]$. Moreover, the participants in the internal focus group reported that they mostly focused on their hand or fingers while throwing the dart in the acquisition phase and retention test. However, 25\% and $8.3 \%$ of them reported that they focused on the dartboard in the acquisition phase or the retention test, respectively. In addition, all the participants in the internal focus group reported that they have focused on their hand or fingers while throwing the dart in the transfer test. In the acquisition phase and the retention test, almost half of the participants in the control group who received no focus instruction reported that they focused on the dartboard or path of the dart and half of them reported they focused on the hand and fingers. However, in the transfer test, only $16.7 \%$ of the participants reported that they focused on the dartboard and $83.3 \%$ of them reported that they focused on the hand and fingers. Similar to those in external and internal focus groups, the participants in the control group possibly tried to freeze more degrees of freedom during the transfer test and therefore focused more on their hand and fingers $[6,15]$. These results are in accordance with those of Abdollahipour et al. [1,2] and provide support for the OPTIMAL theory of motor learning [27].

Beyond the mechanisms underlying the effects of adopting an external focus of attention on motor learning, Wulf and Lewthwaite [27] suggested that adopting an external focus of attention results in promoting focus of the learners on the task goal and this directly connects goals and actions, enhancing goal-action coupling. Adopting an external focus of attention facilitates efficient switching from the default mode network to relevant motor networks, whereas an internal attentional focus impedes this 
process [27]. In the OPTIMAL theory, performing under external attentional focus conditions is presumed to facilitate functional connectivity, that is, task-specific neural connections across distinct brain regions that are seen in skilled performers [1, 2]. Lack of a clear task focus (e.g., internal focus) would impede switching to task-related functional networks or goal-action coupling.

Generally, the results of this study confirm these propositions. The present findings have some practical implications. These results suggest that coaches, trainers and physical educators could optimize learning new motor skills in novices by adopting an external focus of attention during practice. Finally, the present study has some limitations. First, in this study the kinematic patterns of arm throwing were not evaluated. Investigating the effects of adopting an external focus of attention on the learning of movement patterns can help to understand better the process of motor learning influenced by different attentional strategies. Therefore, it is recommended to investigate this issue in future research. Second, the present study sample consisted of male students. Including female subjects to the future research would allow us to determine gender differences in attentional strategies used by individuals to facilitate motor learning.

In conclusion, the results of this study demonstrated that adopting an external focus led to a better motor learning and promoted a focus on the task goal in comparison to an internal focus of attention. Based on the OPTIMAL theory, adopting an external focus of attention reduces self-focus, directs attention to the task goals, and connects goals and actions, e.g., goal-action coupling [27].

\section{Conflict of interest: Authors state no conflict of interest.}

\section{References}

1. Abdollahipour R., Land W.M., Cereser A., Chiviacowsky S. (2019) External relative to internal attentional focus enhances motor performance and learning in visually impaired individuals. Disabil. Rehabil., 8: 1-10.

2. Abdollahipour R., Nieto M.P., Psotta R., Wulf G. (2017) External focus of attention and autonomy support have additive benefits for motor performance in children. Psychol. Sport Exerc., 32: 17-24.

3. Al-Abood S.A., Davids K. Bennett S.J. (2001) Specificity of task constraints and effects of visual demonstrations and verbal instructions in directing learners. J. Mot. Behav., 33: 295-305.

4. Badami R., VaezMousavi M., Wulf G., Namazizadeh M. (2011) Feedback after good trials enhances intrinsic motivation. Res. Q. Exercise Sport, 82: 360-364.

5. Badami R., VaezMousavi M., Wulf G., Namazizadeh M. (2012) Feedback about more accurate versus less accurate trials: Differential effects on self-confidence and activation. Res. Q. Exercise Sport, 83: 196-203.

6. Bernstein N.A. (1967) The Coordination and Regulation of Movement. London: Pergamon Press.

7. Chiviacowsky S. (2014) Self-controlled practice: Autonomy protects perceptions of competence and enhances motor learning. Psychol. Sport Exerc., 15: 505-510.

8. Chiviacowsky S., Wulf G. (2002) Self-controlled feedback: Does it enhance learning because performers get feedback when they need it? Res. Q. Exercise Sport, 73: 408-415.

9. Chiviacowsky S., Wulf G. (2007) Feedback after good trials enhances learning. Res. Q. Exercise Sport, 78: 40-47.

10. Chiviacowsky S., Wulf G., Laroque de Medeiros F., Kaefer A., Tani G. (2008) Learning benefits of self-controlled knowledge of results in 10-year old children. Res. Q. Exercise Sport, 79: 405-410.

11. Chiviacowsky S., Wulf G., Wally R., Borges T. (2009) KR after good trials enhances learning in older adults. Res. Q. Exercise Sport, 80: 663-668.

12. Chiviacowsky S., Wulf G., Lewthwaite R. (2012) Selfcontrolled learning: the importance of protecting perceptions of competence. Frontiers in Psychology, 3, Article 458.

13. Chua L.-K., Dimapilis M.K., Iwatsuki T., Abdollahipour R., Lewthwaite R., Wulf G. (2019) Practice variability promotes an external focus of attention and enhances motor skill learning. Hum. Mov. Sci., 64: 307-319.

14. Ghorbani S. (2019) Motivational effects of enhancing expectancies and autonomy for motor learning: An examination of the OPTIMAL theory. J. Gen. Psychol., 146(1): 79-92.

15. Ghorbani S., Bund A. (2017) Throwing skills: Analysis of movement phases in early motor learning. Percept. Motor Skills, 124(2): 502-513.

16. Hadler R., Chiviacowsky S., Wulf G., Gomes Schild J.F. (2014) Children's learning of tennis skills is facilitated by external focus instructions. Motriz, 20(4): 418-422.

17. Lewthwaite R., Chiviacowsky S., Drews R., Wulf G. (2015) Choose to move: The motivational impact of autonomy support on motor learning. Psychon. Bull. Rev., 22: 1383-1388.

18. Lohse K.R., Sherwood D.E., Healy A.F. (2010) How changing the focus of attention affects performance kinematics, electromyography in dart throwing. Hum. Mov. Sci., 29: 542-555.

19. McKay B., Wulf G. (2012) A distal external focus enhances novice dart throwing performance. Int. J. Sport Exerc. Psychol., 10(2): 149-156.

20. Saemi E., Porter J.M., Ghotbi-Varzaneh A., Zarghami M., Maleki F. (2012) Knowledge of results after relatively good trials enhances self-efficacy and motor learning. Psychol. Sport Exerc., 13: 378-382. 
21. Schorer J., Jaitner T., Wollny R., Fath F., Baker J. (2012) Influence of varying focus of attention conditions on dart throwing performance in experts and novices. Exp. Brain Res., 217(2): 289-297.

22. Schwab S., Rein R. Memmert D. (2019) "Kick it like Ronaldo": a cross-sectional study of focus of attention effects during learning of a soccer knuckle ball free kick technique. German J. Exerc. Sport Res., 49: 91.

23. van Abswoude F., Nuijen N.B., van der Kamp J., Steenbergen B. (2018) Individual Differences Influencing Immediate Effects of Internal and External Focus Instructions on Children's Motor Performance, Res. Q. Exercise Sport, 89(2): 190-199.

24. Wulf G. (2013). Attentional focus and motor learning: A review of 15 years. Int. Rev. Sport Exerc. Psychol., 6: 77-104.

25. Wulf G., Chiviacowsky S., Cardozo P. (2014) Additive benefits of autonomy support and enhanced expectancies for motor learning. Hum. Mov. Sci., 37: 12-20.

26. Wulf G., Chiviacowsky S., Schiller E., Ávila L.T. (2010) Frequent external-focus feedback enhances learning. Frontiers in Psychology, 1, Article 190.
27. Wulf G., Lewthwaite R. (2016) Optimizing performance through intrinsic motivation and attention for learning: the OPTIMAL theory of motor learning. Psychon. Bull. Rev., 23: 1382-1414.

28. Wulf G., Su J. (2007) External focus of attention enhances golf shot accuracy in beginners and experts. Res. $Q$. Exercise Sport, 78: 384-389.

29. Wulf G., Lewthwaite R. Cardozo P., Chiviacowsky S. (2018) Triple play: Additive contributions of enhanced expectancies, autonomy support, and external attentional focus to motor learning. Q. J. Exp. Psychol., 71(4): 824-831.

30. Ziv G., Ochayon M., Lidor R. (2019) Enhanced or diminished expectancies in gold putting - which actually affects performance? Psychol. Sport Exerc., 40: 82-86.

\section{Received 29.07.2019 \\ Accepted 12.11.2019}

(C) University of Physical Education, Warsaw, Poland 